Ann. Biol. anim. Bioch. Biophys., 1979, 19 (3 B), 895-898.

\title{
Corrélations motrices gastro-intestinales chez la poule et la pintade
}

\author{
par M. ROCHE, M. SALIMI*, F. SHAKERI* \\ I.N.S.A., Physiologie-Pharmacodynamie (Bât. 406), \\ 20, avenue Albert-Einstein, 69621 Villeurbanne, France \\ * Faculty of Veterinary Medicine, Physiology and Pharmacology, \\ P. O. Box 3262 Téhëran, Iran.
}

Summary. Interaçtion of gastric and intestinal motility in conscious hens and guinea hens.

Electrical activity was recorded from chronically implanted electrodes in the smooth muscle layers of the gastro-intestinal tract. It was analysed for a long time ( 8 months) in relation to food intake and dark-light schedule.

Two major patterns of electrical activity were identified in the intestine. The first consisted of spike bursts that propagated aborally from the stomach or orally from the ileum. Aborad propagation was induced by the stomach and orad propagation induced an inhibitory effect on the stomach. The second pattern was aborally propagated periods of repetitive spike bursts. As they occurred, there was a reduction or a cessation of gastric spiking activity.

\section{Introduction.}

Des recherches antérieures (Roche, 1973, 1974 ; Roche et Decerprit, 1977) ont mis en évidence les caractéristiques essentielles du profil moteur du tractus digestif des Oiseaux, à savoir : 1) la grande variabilité de l'activité gastro-intestinale en fonction des conditions d'élevage et de la maturité sexuelle, 2) l'existence de nombreux antipéristaltismes intestinaux. Les ondes lentes sont absentes au niveau gastrique ef ne sont présentes que pendant 3 p. 100 du temps d'enregistrement sur le duodénum.

L'objet du présent travail est de préciser les modalités de coordination existant entre l'estomac musculaire et l'intestin chez la Poule (Gallus Gallus) et la Pintade (Numida Meleagris) porfeuses d'électrodes digestives à demeure.

\section{Techniques expérimentales et matériel utilisés.}

Les résultats présentés ont éfé obtenus sur 12 Poules et 2 Pintades femelles élevées dès l'âge de 6 semaines dans des cages individuelles autorisant des en registrements continus $24 \mathrm{~h} / 24$. Elles sont soumises à un éclairement słandard de $12 \mathrm{~h} / 24$ et elles disposent ad libitum d'une alimentation équilibrée, présentée sous forme de farine. Les électrodes 
au nickel-chrome (diamètre $120 \mu$; longueur $65 \mathrm{~cm}$ ) sont dénudées à leur extrémité et implantées à demeure sur l'estomac musculaire ef l'intestin à $5,10,50$ et $80 \mathrm{~cm}$ du pylore. Les enregistrements sont réalisés en continu 3 jours par semaine pendant 8 mois à l'aide d'un polygraphe Alvar XII Pistes (constante de temps $0,1 \mathrm{sec}$.). L'activité élecfrique de deux niveaux d'implantation sélectionnés est, après amplification, sommée par périodes de $20 \mathrm{sec}$. à l'aide d'un intégrateur linéaire (Latour, 1973) relié à un en registreur potentiométrique (PM 8222, Phillips). La branche gastrique du nerf pneumogastrique est sectionnée chez 3 Poules.

\section{Résultats.}

Chez la Poule ef la Pintade l'activité électrique de l'estomac musculaire est continue excepté quelques courtes inhibitions ef un rythme moyen réduit lors de la nuit (fig. 1). L'activité électrique de l'infestin comprend d'une part de courtes salves de potentiels de pointe isolées ou groupées par 3-4 et propagées rapidement $(3 \mathrm{à} 10 \mathrm{~cm} / \mathrm{sec}$.) dans le sens aboral ou oral, d'autre part des salves de potentiels successives pendant 5 à $8 \mathrm{mn}$ comparables par quelques aspects à la phase régulière du complexe myoélectrique des Mammifères et propagées lentement $(3 \mathrm{~cm} / \mathrm{mn})$ le long de l'intestin.

\section{ESTOMAC}

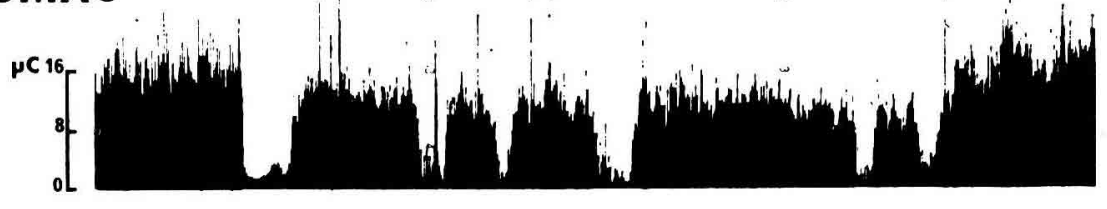

\section{DUODENUM + $10 \mathrm{~cm}$}

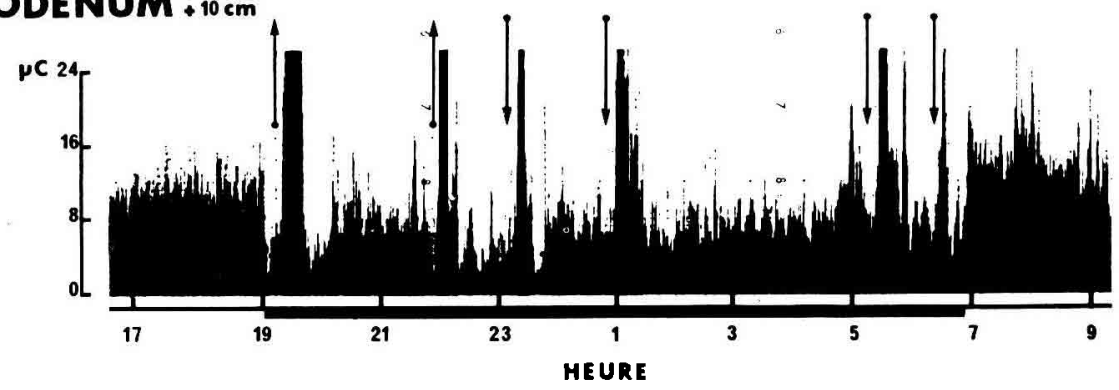

FIG. 1. - Sommation par périodes de 20 s de l'activifé électrique (exprimée en microcoulomb, $\mu \mathrm{C}$ ) de l'estomac musculaire ef du duodénum $(10 \mathrm{~cm}$ du pylore) chez la Poule. Les périodes continues de salves de potentiels de pointe propagées le long de l'intestin dans le sens aboral $(\downarrow)$ à partir du pylore ou oral $(\uparrow)$ à partir de l'iléon correspondent à une inhibition de l'activité gastrique.

Les salves de potentiels à propagation aborale rapide sont liées à l'activité gastrique puisque 1) elles apparaissent obligatoirement entre la mise en jeu des muscles intermédiaires et des màchoires de l'estomac musculaire, 2) elles disparaissent avec la motricité gastrique, 3) l'accélération gastrique due à la prise de nourriture favorise les salves de potentiels isolées aux dépens des groupes de 3-4 salves. Cependanf, la 
dépendance de l'intestin vis-à-vis de l'estomac n'est pas totale : après in hibition par le sulfate d'atropine $\left(1 \mathrm{mg} \cdot \mathrm{kg}^{-1} \mathrm{iv}\right)$ l'activité électrique du duodénum réapparait 5 à $10 \mathrm{mn}$ avant celle de l'estomac.

Les salves de potentiels antipéristaltiques sont issues de la jonction iléo-cæcale. Leur durée varie entre 2 et $4 \mathrm{sec}$. chez la Poule, 20 et $45 \mathrm{sec}$. chez la Pintade. Elles sont contrôlées par le territoire gastrique puisque 1) elles nécessitent un rythme gastrique réduit au dizième de sa fréquence moyenne, 2) les inhibitions gastriques observées la nuit ou lors du jeône facilitent l'activité antipéristaltique de l'intestin, 3) la vagotomie sélective gastro-intestinale inhibe à 80 p. 100 la motricité gastrique et peut augmenter jusqu'à 200 p. 100 le nombre des antipéristaltismes intestinaux, 4) chez la Poule, des phases de 15 à 40 salves de potentiels antipéristaltiques successives n'apparaissent qu'après une inhibition préalable totale de l'estomac pendant 10 à $30 \mathrm{mn}$ (fig. 1). Par contre, chez la Pintade la propagation orale des salves de potentiels isolées exerce un effet inhibiteur sur l'estomac.

La propagation aborale lente des phases de salves de potentiels successives est marquée par une réduction de l'activité gastrique, 1 à 2 fois par nycthémère elles débutent près du pylore et inhibent totalement l'estomac.

\section{Discussion et conclusion.}

La coordination gastro-intestinale mise en évidence chez les Mammifères (Ruckebusch, 1975) a été suggérée par Duke, Kostuch ef Evanson (1975) chez le Dindon. Les présents résultats soulignent que l'estomac impose à l'intestin la propagation aborale rapide de salves de potentiels correspondant à une contraction importante de la paroi musculaire (Roche et Ruckebusch, 1978), ceci devant faciliter le transit gastro-duodénal des ingesta. Les antipéristaltismes intestinaux nécessitent une activité gastrique réduite et inhibent l'estomac lors de leur propagation, ceci s'expliquant par la nécessité d'un relâchement gastrique lors du reflux des ingesta de l'intestin vers l'estomac. Enfin, les phases de contraction soutenue du duodénum exercent un effet inhibiteur sur l'estomac, ce qui réduit ou stoppe le flux des ingesta quand la capacité duodénale est minimum. L'influence prépondérante de l'estomac sur l'intestin peut résulter de l'absence de régulation de l'activité électrique rapide de l'intestin par les ondes lentes.

Commission CNERNA Digestion-Absorption/Association des Physiologistes, Paris 5-6 octobre 1978.

Remerciements. - Ce travail a été partiellement financé par le Conseil de recherches scientifiques d'Iran, Ministère des Sciences et de l'Enseignement supérieur.

\section{Références}

DUKE G. E., KOSTUCH T. E., EVANSON O. A., 1975. Gastroduodenal electrical activity in furkeys. Am. J. Digest. Diseases, 20, 1047-1058.

LATOUR A., 1973. Un dispositif simple d'analyse quantitative de l'électromyogramme intestinal chronique. Ann. Rech. vét., 4, 347-353. 
ROCHE M., 1973. Motricité digestive des oiseaux. Etude électromyographique chez le poulet. Cah. Med. vét., 2, 117-118.

ROCHE M., 1974. Motricité gastro-intestinale du poulet. Ann. Rech. vét., 5, 295-309.

ROCHE M., DECERPRIT J., 1977. Contrôles hormonal et nerveux de la motricité du tractus digestif de la poule. Ann. Rech. vét., 8, 25-40.

ROCHE M., RUCKEBUSCH Y., 1978. A basic relationship between gastric and duodenal motilities in chickens. Am. J. Physiol., 235 (sous presse).

RUCKEBUSCH Y., 1975. Inferaction of duodenal and antral activity in sheep and dogs. Proc. Physiological Society, J. Physiol. London, 254, 79-80 p. 